\title{
A Mathematical Model of Herschel- Bulkley fluid through an overlapping stenosis
}

\author{
K.MaruthiPrasad ${ }^{1}$, BhuvanaVijaya $^{1,}$ C.Umadevi ${ }^{2}$, \\ 1. Department of Mathematics, GITAM University, Hyderabad Campus, A.P, India. \\ 1. Department of Mathematics, JNTU College of Engineering, Anantapur, A.P, India \\ 2. Department of Mathematics, TKR College of Engineering, Hyderabad, A.P. India
}

\begin{abstract}
The steady flow of Herschel-Bulkely fluid through a tube having overlapping stenosis has been investigated. Assuming the stenosis is to be mild, the flow equations have been linearised and the expressions for the resistance to the flow and wall shear stress have been studied. It is found that the resistance to flow increases with the height of the stenosis, power law index, wall shear stress andyield stress but decreases with stress ratio parameter. It is proved that the Hershcel-Bulkely fluid is more appropriate model to the stenosis than the Newtonian fluid.
\end{abstract}

Key Words:Herschel-Bulkely fluid, resistance to the flow, power law index, stress ratio parameter, stenosis

\section{Introduction}

Healthcare problems are apparently concerned by the people in these days. For over centuries, cardiovascular diseases have been noticed as one of major illnesses where numerous people suffer from them. The cardiovascular system consists of the heart and blood vessels which plays an important role in transportation, protection and regulation of human body. A pressure gradient is produced as the heart pumps, so that blood will flow through the body. Among the cardiovascular diseases, the familiar ones such as stroke and atherosclerosis are closely related to abnormality, disorder and malfunction of blood flow characteristics in human body, Owing to this, blood flow related problems have shown significant interest for biomedical researchers.

Stenosis or arteriosclerosis is the abnormal and unnatural growth in the arterial wall thickness that develops at various locations of the cardiovascular system under diseased conditions. This may be caused by unhealthy living conditions such as exposure to tobacco smoke, lack of physical activity and improper dietary habits. It is always followed by the serious changes in blood flow, pressure distribution, wall shear stress and flow resistance.

Once arterial stenosis occurs, atherosclerotic plaques would protrude into lumen of blood vessels. Consequently, resistance is increased; hence blood flow is insufficient to reach every cell and this resists nutrient supplement. These could lead to wide spread of health disorders which may worsen various illnesses. To more serious extent, these abnormalities in blood flow could contribute substantial fatal health risks.

In view of this, several authors have considered various mathematical models for flows through stenosed/constricted ducts (young(1968), Lee and Fung(1970), Shukla et al. (1980), Chaturani and Samy (1986), Radhakrishnamachary and SrinivasRao(2007).

In all these mathematical studies, blood has been considered as Newtonianfulid. But Majhi and Nair (1996) suggested that blood behaves like a non-Newtonian fluid under certain conditions. It is also realized that the Herschel-Bulkley model is a better model than Casson's model (Blair and Spammer (1974)). Further, in small diameter tubes blood behaves like a Heerschel-Bulkley fluid rather than power law and Bingham fluids (Chaturani and Samy (1985)). However, all theseinvestigations considered the effect of single stenosis but, the constrictions may develop in series (multiple stenoses) or may be of irregular shapes or overlapping. Chakravarthy and Mandal (1994) studied effects of overlapping stenosis on arterial flow problem analytically by assuming the pressure variation only along the axis of tube. Maruthi Prasad and Radhakrishnamacharya (2008) investigated the flow of Herschel-Bulkley fluid through an inclined tube of non uniform cross section with multiple stenoses. Layek et al (2009) investigated the effects of overlapping stenosis on flow characteristics considering the pressure variation in both the radial and axial directions of the arterial segment under consideration and Srivastava et al. (2007) studies the blood flow through a overlapping stenoses assuming that the flowing blood is represented by two layered macroscopic two-phase model.

An attempt is made in the present investigation to explore the effects of an overlapping stenosis on the flow characteristics of blood taking into account the flowing blood is to be treated as an incompressible nonNewtonian (Herschel-Bulkley) fluid. The arterial wall segment is considered to be rigid as well as deformable. The wall in the vicinity of the stenosis is usually relatively rigid when stenosis develops in human vasculature. 
The artery length is considered to large enough as compared to the radius, so that the entrance, end and special wall effects can be neglected.

\section{Mathematical Formulation}

Consider the assymmetric, steady flow of H-B fluid through a tube of circular cross section with an overlapping stenosis at the positions as shown in fig-1. The radius of the cylindrical tube is assumed as(Chakravarty and Mandal (1994); Layek et al.(2009);Srivastava et al.(2010)

$h=\frac{R(z)}{R o}=1-3 / 2\left(\frac{\delta}{R o L o^{4}}\left[11(z-d) L o^{3}-47(z-d)^{2} L o^{2}+72(z-d)^{3} L o-36(z-d)^{4}\right], d \leq z \leq d+L o\right.$, $=1$, otherwise.

Where $R(Z)$ is the radius of the tube with stenosis,$R_{0}(Z)$ is the radius of the tube without stenosis, $R_{p}$ is the radius of the plug flow region, $L_{0}$ is the length of the stenosis and $d$ is its location, $\delta$ is the maximum height of the stenosis. Projection of stenosis at the two positions isdenoted by $Z$ as $Z=d+L_{o} / 6, Z=d+5 L_{o} / 6$, respectively. The critical height is taken as $3 \delta / 4$ at $Z=d+L_{o} / 2$, from the origin.

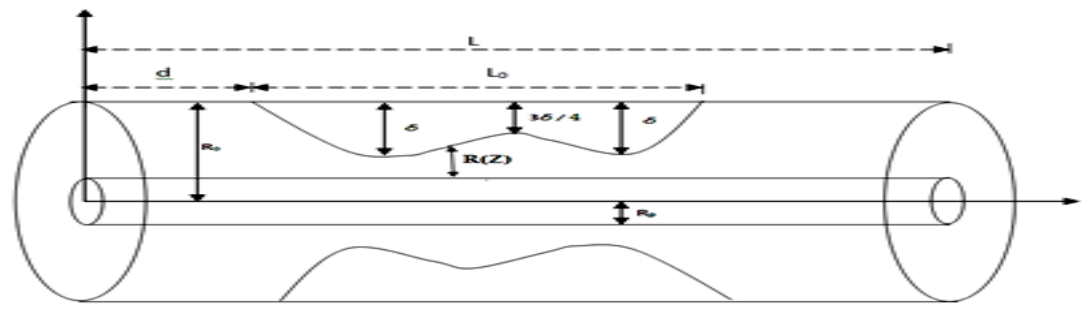

Fig. 1: Geometry of a uniform tube of circular cross section with overlapping stenosis

The general equations, considering a mild stenosis in an artery of circular cross section under the conditions

$\frac{1}{r} \frac{\partial}{\partial r}\left(r \tau_{r z}\right)=-\frac{\partial p}{\partial z}$

Where $\tau_{\mathrm{rz}}$ is the shear stress for H-B field fluid, is given by

$\tau_{\mathrm{rz}}=\left(\frac{-\partial u}{\partial r}\right)^{n}+\tau_{\mathrm{o}}$, if $\tau_{\mathrm{rz}} \geq \tau_{\mathrm{o}}$

$\frac{\partial u}{\partial r}=0$, if $\tau_{\mathrm{rz}}<\tau_{\mathrm{o}}$

Where $(r, z)$ are cylindrical polar co-ordinates withz measured along the tube axis and $r$ is measured along the normal to the axis of the tube. ' $P$ ' is pressure, ' $\tau_{\mathrm{rz}}$ ' is shear stress and ' $\tau_{\mathrm{o}}$ ' is yield stress and ' $u$ is the velocity of the fluid.

The boundary conditions are

$\begin{array}{ll}\text { (i) } & \tau_{\mathrm{rz}} \text { is finite at } r=0 \\ \text { (ii) } & u=0, \text { at } r=h(z) \\ \text { (iii) } & \frac{\partial u}{\partial r}=0 \text { at } r=0 \text { if } \tau_{\mathrm{rz}}<\tau_{\mathrm{o}}\end{array}$

\section{Solution}

The solution of eq.(2) under the boundary conditions (4),(5)and (6), the velocity is obtained as

$$
u=\frac{h^{(k+1)} p^{k}}{2^{k}(k+1)}\left\{\left(1-\frac{2 \tau_{o}}{h p}\right)^{k+1}-\left(\frac{r}{h}-\frac{2 \tau_{o}}{h p}\right)^{k+1}, \quad r_{0} \leq r \leq h\right.
$$

Where $p=-\frac{\partial p}{\partial z} \cdot k=\frac{1}{n}$

Using the boundary condition (3), the upper limit of the plug flow region is obtained as $r_{0}=2 \tau_{0} / p$

and using the condition $\tau_{\mathrm{rz}}=\tau_{\mathrm{h}}$ at $r=h$,

$\frac{r_{o}}{h}=\frac{\tau_{o}}{\tau_{h}}=\tau, 0<\tau<1$

$u_{p}=\frac{h^{(k+1)} p^{k}}{2^{k}(k+1)}\left(1-\frac{2 r_{o}}{h p}\right)^{k+1}$,

for $0 \leq r \leq r_{\mathrm{o}}$

The flow plug $Q$ is defined as

$Q=2\left[\int_{0}^{r_{0}} u_{p} r d r+\int_{r_{0}}^{h} u r d r\right]$

From eq.(11), we get

$Q=A\left[(k+2)(k+3)\left(1-\frac{r}{h}\right)^{k+1}-2(k+3)\left(1-\frac{r_{o}}{h}\right)^{k+2}+2\left(1-\frac{r_{o}}{h}\right)^{k+3}\right]$ 
Where $A=\frac{h^{(k+3)} p^{k}}{2^{k}(k+1)(k+2)(k+3)}$

From eq (12),

$\frac{d p}{d z}=-p=\frac{2 Q^{\frac{1}{k}}[(k+1)(k+2)(k+3)]^{\frac{1}{k}}}{h^{1+\frac{3}{k}}\left\{(k+2)(k+3)(1-\tau)^{k+1}-2(1-\tau)^{k+2}(k+2+\tau)\right\}^{\frac{1}{k}}}$

The pressure drop $\Delta p$ across the stenosis between $z=0$ to $z=L$ is obtained by integrating eq.(13), as $\Delta p=\int_{0}^{L} \frac{d p}{d z} d z$

$\Delta p=\int_{0}^{L} \frac{2 Q^{\frac{1}{k}}[(k+1)(k+2)(k+3)]^{\frac{1}{k}}}{h^{1+\frac{3}{k}}\left\{(k+2)(k+3)(1-\tau)^{k+1}-2(1-\tau)^{k+2}(k+2+\tau)\right\}^{\frac{1}{k}}} d z$

Introducing the following non-dimensional quantities

$\bar{Z}=\frac{z}{L}, \bar{\delta}=\frac{\delta}{R_{0}}, \bar{R}(z)=\frac{R(z)}{R_{0}}, \quad \bar{P}=\frac{P}{\left(\mu U L / R_{0}^{2}\right)}$,

$\overline{\tau_{0}}=\frac{\tau_{0}}{\mu\left(\frac{U}{R_{0}}\right)}, \quad \overline{\tau_{r z}}=\frac{\tau_{r z}}{\mu\left(\frac{U}{R_{0}}\right)}, \quad \bar{Q}=\frac{Q}{\pi R_{0}^{2} U}$

In eq.(15), we finally get (after dropping the bars)

$\Delta p=\int_{0}^{1} \frac{2 Q^{\frac{1}{k}}[(k+1)(k+2)(k+3)]^{\frac{1}{k}}}{h^{1+\frac{3}{k}}\left\{(k+2)(k+3)(1-\tau)^{k+1}-2(1-\tau)^{k+2}(k+2+\tau)\right\}^{\frac{1}{k}}} d z$

The resistance to the flow, $\lambda$, is defined by

$\lambda=\frac{\Delta p}{Q}=\frac{1}{Q} \int_{0}^{l} \frac{2 Q^{\frac{1}{k}}[(k+1)(k+2)(k+3)]^{\frac{1}{k}}}{h^{1+\frac{3}{k}}\left\{(k+2)(k+3)(1-\tau)^{k+1}-2(1-\tau)^{k+2}(k+2+\tau)\right\}^{\frac{1}{k}}} d z$

the pressure drop in the absence of $\operatorname{stenosis}(h=1)$ is denoted by $\Delta p_{N}$, is obtained from eq.(16)

$\Delta p_{N}=\int_{0}^{1} \frac{2 Q^{\frac{1}{k}}[(k+1)(k+2)(k+3)]^{\frac{1}{k}}}{\left\{(k+2)(k+3)(1-\tau)^{k+1}-2(1-\tau)^{k+2}(k+2+\tau)\right\}^{\frac{1}{k}}} d z$

The resistance to the flow in the absence of stenosis is denoted by $\lambda_{N}$ is obtained from eq.(18), as

$\lambda_{N}=\Delta p_{N} / Q=\quad \frac{1}{Q} \int_{0}^{1} \frac{2 Q^{\frac{1}{k}}[(k+1)(k+2)(k+3)]^{\frac{1}{k}}}{\left\{(k+2)(k+3)(1-\tau)^{k+1}-2(1-\tau)^{k+2}(k+2+\tau)\right\}^{\frac{1}{k}}} d z$

the normalized resistance to the flow denoted by

$\bar{\lambda}=\lambda / \lambda_{N}$

\section{Results And Analysis}

The effects of various parameters on the resistance to the flow $(\bar{\lambda})$ and shear stress $\left(\tau_{\mathrm{h}}\right)$ acting on the wall are computed numerically by taking $L_{0}(\mathrm{~cm})=1, L(\mathrm{~cm})=1, \delta=0.05,0.10,0.015,0.02$.

It is observed that the resistance to the flow $(\bar{\lambda})$ increases with height of the stenosis $(\delta)$ but decreases with the stress ratio parameter $\tau$ i.e the ratio between the yield stress and wall shear stress $\left(\frac{\tau_{o}}{\tau_{h}}=\tau\right)($ Fig.2).

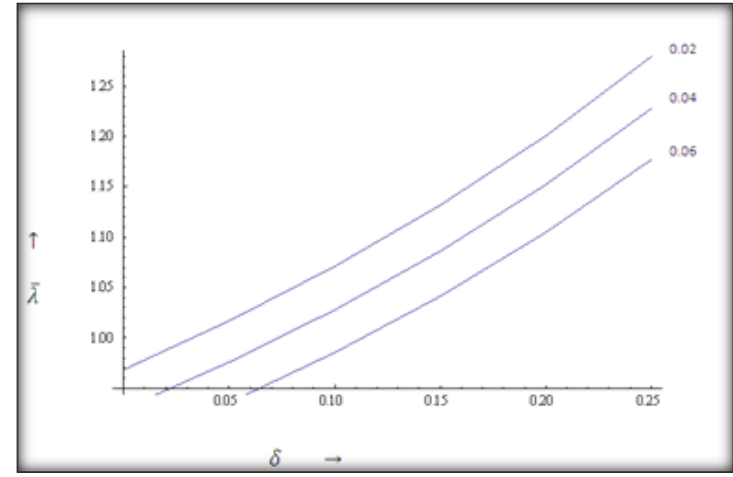

Fig.2: Variation of impedance $\bar{\lambda}$ with $\delta$ for different $\tau\left(d=0.2, L=1, L_{0}=1, k=2000, q=0.1\right)$ 


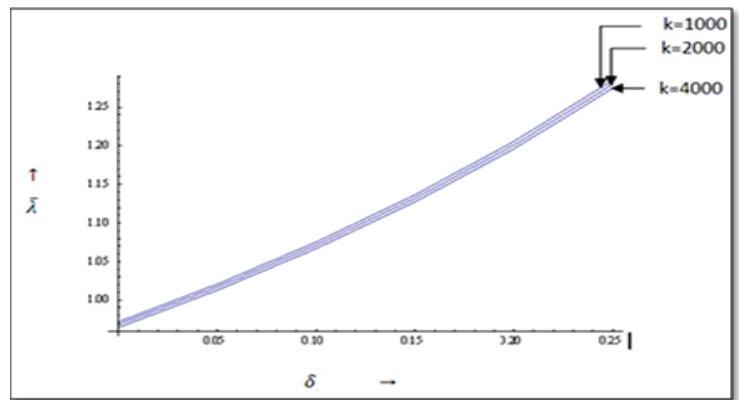

Fig.3: Variation of impedance $\bar{\lambda}$ with $\delta$ for different $k\left(d=0.2, L=1, L_{0}=1, \tau=0.02, q=0.1\right)$ It is noticed from Fig. 3 that the resistance to the flow or impedance $\bar{\lambda}$ increases with the height of the stenosis and also with the power law index $\left(n=\frac{1}{k}\right)$ i.e. the resistance increases with non-Newtonian character of the fluid.

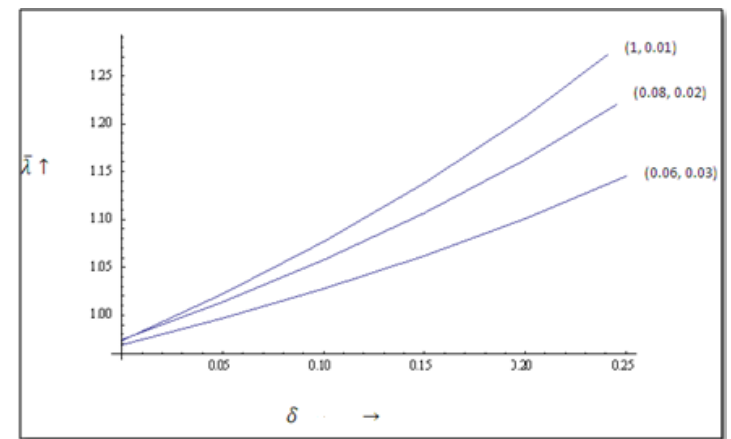

Fig.4: Variation of impedance with $\delta$ for different values of $L$ and $\tau\left(d=0.2, L_{0}=1, k=2000, q=\right.$ 0.1)

It is observed from the fig. 4 that the resistance to the flow (impedance) $\bar{\lambda}$ with the length of the tube $L$ and decreases with stress ratio parameter $\tau\left(=\frac{\tau_{o}}{\tau_{h}}\right)$. The resistance to the flow increases with the wall shear stress $\left(\tau_{h}\right)$ and decreases with stress ratio parameter (Fig 5-6)

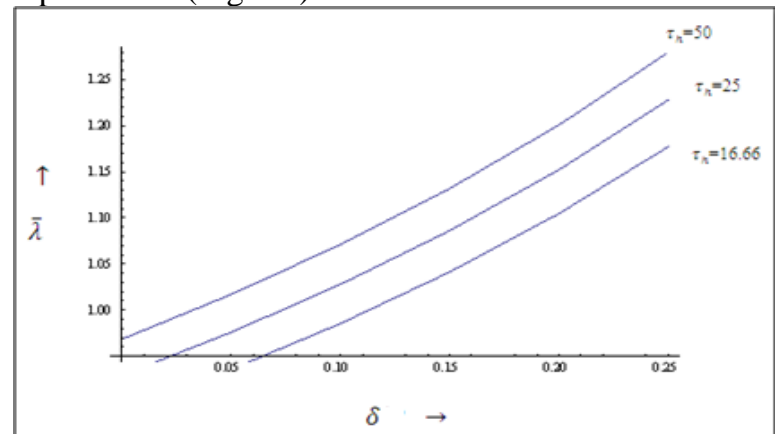

Fig.5: Variation of impedance $\bar{\lambda}$ with $\delta$ for different $\tau_{h}\left(d=0.2, L_{0}=L=1, k=2000, q=0.1 \tau_{0}=5\right)$

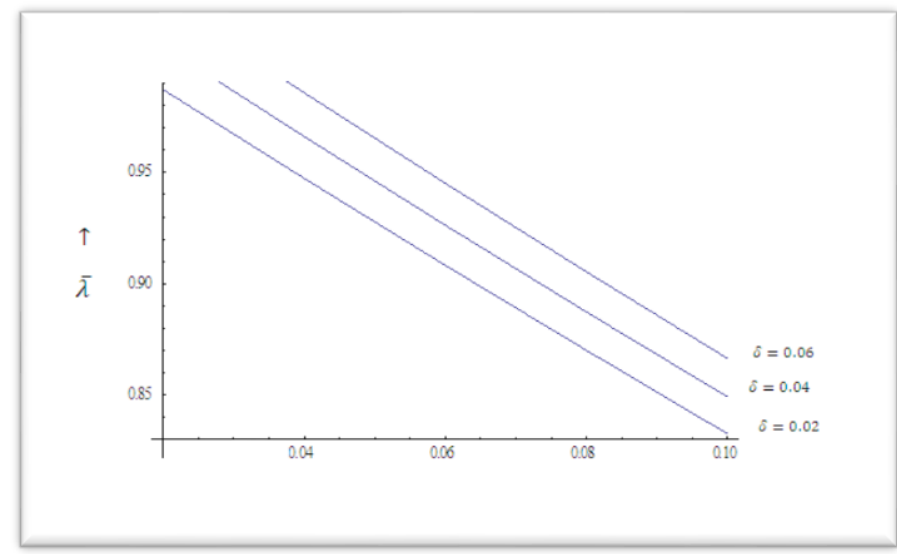

Fig.6: Variation of impedance $\lambda$ with $\tau$ for different $\delta\left(d=0.2, L_{0}=1, k=2000, q=0.1\right)$ 
It can also be observed from Fig. 7 that the resistance to the flow (impedance) $\bar{\lambda}$ decreases with stress ratio parameter $\tau\left(=\frac{\tau_{o}}{\tau_{h}}\right)$, and increases with height of the stenosis and power law index $\left(n=\frac{1}{k}\right)$

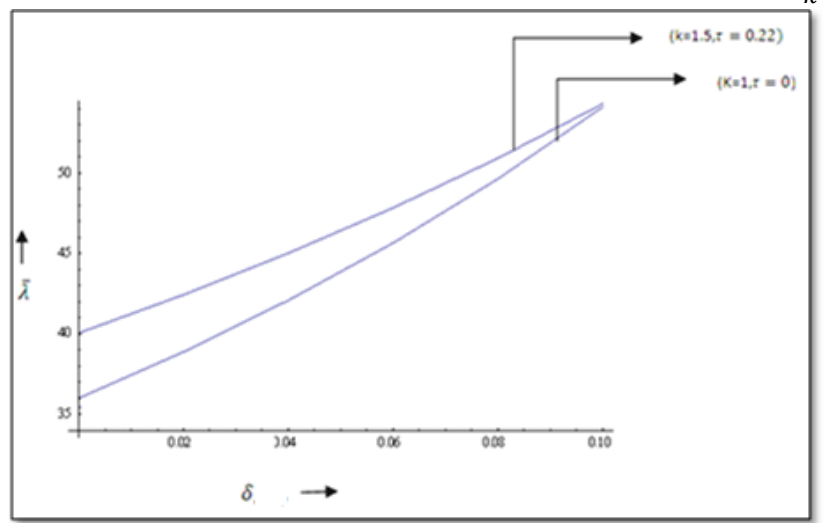

Fig.7: Variation of impedance $\bar{\lambda}$ with $\delta$ for $\tau$ and $k\left(d=0.2, L=L_{0}=1, q=0.1\right)$

The Herschel - bulkely fluid behaves as Newtonian fluid when power law index $n=1$ and $\tau_{0}=0$ and it acts as a power-law fluid when $n=n$ and yield stress $\tau_{0}=0$.

It can also be observed from the Fig.7that the resistance to the flow increases with the yield stress. It is interesting to note that the resistance of the H-B fluid is higher than the Newtonian fluid because of yield stress.

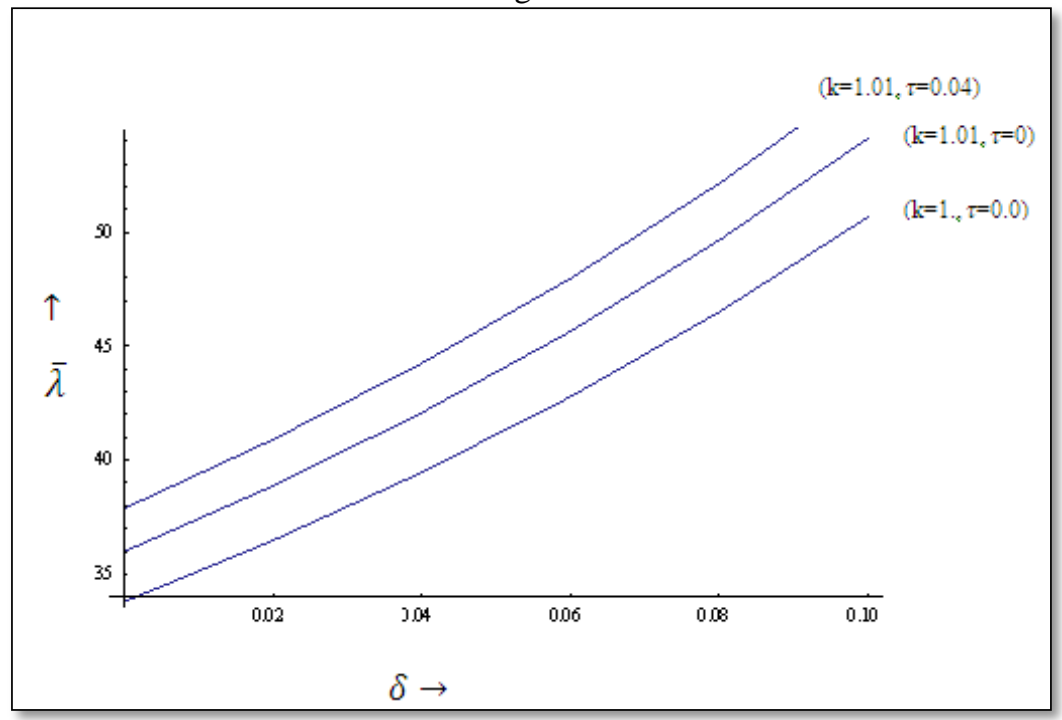

Fig.8: Variation of impedance $\bar{\lambda}$ with $\delta$ for different fluids $\left(d=0.2, L=1, L_{0}=1, q=0.1\right)$

It is quite interesting to observe from the Fig.8that asthe resistance to the flow increases with the height of the stenosis and it is clear that the resistance of the flow is higher in Herschel-bulkley fluid comparing with the Newtonian fluid and difference of increase is $2 \%$ (approximately).

\section{Conclusion}

A Mathematical model for the steady flow of Herschel-Bulkely fluid through a tube having overlapping stonosis has been presented. Solutions have been obtained for mild stenosis and it has been shown that the resistance to the flow increases with heights of the stenoses, yield stress and power law index but decreases with stress ratio parameter. The Herschel -Bulkley fluid is more sensitive to stenosis than the power law fluid and Newtonian fluid.

\section{References}

[1] Young, D.F.,1968, Effects of a time-dependent stenosis on flow through tube. Engrg. Ind. Trans ASME. 90, 248-254.

[2] Lee, J.S., Fung,Y.C 1970, Flow in Locally- constricted Tubes and Low RenoldsNumbers,J. Appl.Mech. Trans ASME. 37, 9-16

[3] Shukla, J.B., Parihar,R.S., Rao, B.R.P.,1980, Effects of stenosis on non-Newtonian flow through an artery with mild stenosis,Bull.Math.Biol. 42, 283-294.

[4] Chaturani, P., PonnalagarSamy,R., 1986, Pulsatile flow of Casson's fluid through stenosedarteries with applications to blood flow. Biorheol. 23, 499-511

[5] Radhakrishnamacharya,G., SrinavasRao,P., 2007, Flow of a magnetic fluid through a nonuniform wavy tube, Proc.Nat.Acad.Sci.,India 76(A), in press . 
[6] Majhi,S.N., Ajhi, Nair,V.R.,1996, Pulsatile flow of third grade fluids under body acceleration-modeling blood flow .Int.J.Engg.Sci.32,5,839-846.

[7] Blair, G.W.S., Spanner, D.C.,1974, An introduction to Biorheology.Elsevier., Amsterdam.

[8] Chaturani, P., PonnalagarSamy,R., 1985, A study of non-Newtonian aspects of blood flowthrough stenosed arteries and its applications in arterial diseases .Biorheol.22,521-531.

[9] Chakravarthy,S., Mandal,P.K., 1994, Mathematical modelling of blood flow through an overlapping stenosis .Math.Comput.Model .Vol.19,pp.59-73.

[10 ]MaruthiPrasad,K., Radhakrishnamacharya,G., 2008, Flow of Herschel-Bulkley fluid through an inclined tube of non-uniform cross-section with multiple stenoses. Arch.Mech.0,2,pp.161-172,Warszawa.

[11] Layek,Mukhopadhya, G.C., and Glora,R.S.D.,2009,Unsteadyviscous flow with variousviscosity in a vascular tube with an overlapping constriction. Int.J.Engng. Sci. 47,pp 649-659.

[12] Srivastava, V.P., andRastigi, R., 2010, Blood flow through stenosed catheterized artery: effects of hematocrit and stenosis shape. Comput.Math.Applc.59,1377-1785. 